\title{
Analysis of a production-inventory system with unreliable production facility
}

\author{
Katrien Ramaekers and Gerrit K. Janssens \\ Transportation Research Institute \\ Hasselt University - Campus Diepenbeek \\ Wetenschapspark 5 bus 6 \\ B-3590 Diepenbeek, Belgium \\ e-mail:\{katrien.ramaekers,gerrit.janssens\}@uhasselt.be
}

\begin{abstract}
KEYWORDS
production-inventory system, simulation model, limited information on demand, robustness

\section{Abstract}

This paper tests the robustness of analytical results on performance measures for a production-inventory system with an unreliable production facility. A basic model is considered which involves a single production machine with constant production rate which operates as long as the accumulated inventory is below a pre-set threshold, and is idle otherwise. The demand process follows a compound Poisson distribution and the operating time of a machine until failure as well as the repair time of a failed machine are assumed to be exponentially distributed. A demand that cannot be fully satisfied from existing inventory takes whatever is available and the rest of the demand is lost. Analytical results exist only for the special case where the order sizes are exponentially distributed. In this paper, a simulation model is built to obtain performance measures for the same production-inventory system facing other demand processes. These results are used to test the robustness of the analytical results to changes in the demand process.
\end{abstract}

\section{INTRODUCTION}

Production-inventory models link the production process to the demand process to ensure that shortages are kept at a low level and no excessive inventory is built up. The models vary in terms of the characteristics of the production process, the demand pattern, the customer response to shortages and the inventory review procedures.

In this paper, the production system is considered to be imperfect: machines can fail resulting in disrupted production plans, reduced inventory and shortages. Posner and Berg (1989) derived an explicit closed form solution for the steady state distribution of the inventory level and used this result to compute system performance indices of interest related to service level to customers and machine utilization. They consider a basic model which involves only one production machine with constant production rate which operates as long as the accumulated inventory is below a pre-set threshold, and is idle otherwise. The demand process is compound Poisson and the operating time of a machine until failure as well as the repair time of a failed machine are assumed to be exponentially distributed. A demand that cannot be fully satisfied from existing inventory takes whatever is available and the rest of the demand is lost.

When dealing with slow moving products or products recently introduced to the market, there is not sufficient data to decide on the functional form of the demand distribution function and it is realistic to assume the demand distribution is incompletely known. Limited but not full information might exist, like the range of the demand, the expected value and its variance. We assume the same mean and standard deviation for demand can be obtained by various patterns regarding demand frequency and size.

Posner and Berg (1989) assume a compound Poisson demand process with exponentially distributed order sizes. We test the robustness of their results to changes in the demand process. However, these changes will be chosen in such a way that they do not result in a different mean or standard deviation of the demand distribution. Unfortunately no closed-form formulas exist when the order size does not follow an exponential distribution. Therefore, simulation models have to be used to determine performance measures of these systems.

\section{The PRODUCTION-INVENTORY MODEL}

In this section, the production-inventory system that is used by Posner and Berg (1989) is discussed in more detail.

\section{A. Definitions and assumptions}

Following assumptions are made:

- Production is continuous. No individual parts can be identified.

- Production speed is, without loss of generality, assumed to be equal to 1 . This means that the machine outputs 1 unit of product per time unit.

- Production is halted whenever the inventory level reaches $M$, and is resumed when the next order arrives. - The operating time until failure is exponentially distributed. The average failure rate of the machine is the 
inverse of the mean time to failure (MTTF).

$$
\theta=\frac{1}{M T T F}
$$

- The repair time of each machine is exponentially distributed. The average repair rate of the machine is the inverse of the mean time to repair (MTTR).

$$
\sigma=\frac{1}{M T T R}
$$

- The demand process is compound Poisson. Orders arrive according to a Poisson process with rate $\lambda$ and order sizes are i.i.d. random variates exponentially distributed with mean $\mu^{-1}$. Demand that cannot be satisfied, is lost.

\section{B. Single machine model}

Posner and Berg (1989) study a simple system of a single machine which outputs into an inventory. Using the generalized technique of the 'system point' in levelcrossings analysis (Brill and Posner, 1977; Brill and Posner, 1981), Posner and Berg derive an analytical formula from which a number of performance measures can be calculated.

The availability of the machine (fraction of time the machine is engaged in active production), is equal to

$$
A=\alpha\left[\frac{1-e^{-\beta_{1} M}}{\beta_{1}}-\frac{r}{\beta_{2}}\left(1-e^{-\beta_{2} M}\right)\right] .
$$

The fraction of time the machine is down due to failures is equal to $1-A-\gamma$. The machine shutdown rate due to overstocking is equal to $\lambda \gamma=\alpha(1-r)$. The demand rate is equal to $\lambda / \mu$. The production rate is 1 , therefore the overall effective production rate is equal to $A$. The fraction of loss due to shortages therefore is equal to $\lambda / \mu-A$. The fraction of demand satisfied is equal to $\frac{A}{\lambda / \mu}$. The machine failure rate is equal to $\theta A$. The average inventory is equal to

$\alpha\left[\frac{\mu-\beta_{1}}{\lambda \beta_{1}^{2}}\left(1-e^{-\beta_{1} M}\right)-\frac{r\left(\mu-\beta_{2}\right)}{\lambda \beta_{2}^{2}}\left(1-e^{-\beta_{2} M}\right)\right]+M \delta$.

The unknowns in these equations can be calculated from the following equations:

$$
\begin{aligned}
\lambda \alpha^{-1}= & \frac{\mu}{\beta_{1}}\left(1-\frac{r \beta_{1}}{\beta_{2}}\right)+\left(1-\frac{\mu}{\beta_{1}}\right) e^{-\beta_{1} M} \\
& -r\left(1-\frac{\mu}{\beta_{2}}\right) e^{-\beta_{2} M}+\frac{\lambda}{\sigma}\left(\mu-\lambda-\beta_{1}\right) \\
& {\left[\frac{\beta_{2}-\beta_{1}}{\left(\mu-\beta_{1}\right)\left(\mu-\beta_{2}\right)} e^{-\mu M}\right.} \\
\beta_{1}, \beta_{2}= & \left.\mu-\frac{1}{2} \cdot \frac{\lambda}{\lambda+\sigma}-\frac{e^{-\beta_{1} M}}{\mu-\beta_{1}}-\frac{e^{-\beta_{2} M}}{\mu-\beta_{2}}\right] \\
& {[\lambda+\sigma+\theta+\mu} \\
& \left. \pm \sqrt{(\lambda+\sigma+\theta-\mu)^{2}+4 \theta \mu}\right]
\end{aligned}
$$

$$
\begin{aligned}
r= & \frac{\mu-\lambda-\beta_{1}}{\mu-\lambda-\beta_{2}} \cdot \frac{\mu-\beta_{2}}{\mu-\beta_{1}} \\
\gamma= & \frac{\alpha(1-r)}{\lambda} \\
\delta= & \frac{\alpha}{\sigma}\left[\frac{\mu-\lambda-\beta_{1}}{\beta_{1}-\mu}\left(e^{-\mu M}-e^{-\beta_{1} M}\right)\right. \\
& \left.-r \frac{\mu-\lambda-\beta_{2}}{\beta_{2}-\mu}\left(e^{-\mu M}-e^{-\beta_{2} M}\right)\right]
\end{aligned}
$$

\section{Simulation APPROACH}

Because no closed-form formulas exist when the order size does not follow an exponential distribution, simulation models have to be used to determine performance measures of these systems. However, simulation results can be difficult to interpret. Each simulation run leads to an estimate of the model's characteristics and, as a result, these estimates can differ from the corresponding true characteristics of the model. Appropriate statistical techniques must be used to analyze and interpret the simulation experiments. Two main issues will be discussed: output data analysis and common random numbers.

\section{A. Output data analysis}

As mentioned before, a single run of a simulation model can lead to serious errors and poor decisions. In order to obtain a point estimate and confidence interval for a simulation output, several runs of the simulation model using different random numbers are needed. Several methods exist to estimate the outputs confidence intervals. The method we use in this paper is the replication/deletion approach because it is a simple approach that gives good statistical performance (Law, 2007). It can easily be used to estimate several different parameters for the same simulation model and to compare different system configurations.

To validate the simulation model we built, the analytical results of Posner and Berg (1989) are compared to the results of the simulation model with exponentially distributed order sizes. A 95\% confidence interval is constructed for the simulation output.

Suppose we make $n$ replications of the simulation. The independence of replications is accomplished by using different random number series for each replication. Let $Y_{j}$ be the $j$ th replication (for $j=1,2, \ldots, n$ ) of the measure of performance of interest. An unbiased point estimator for the mean $\mu=E(Y)$ is given by

$$
\bar{Y}(n)=\frac{\sum_{j=1}^{n} Y_{j}}{n} .
$$

and an approximate $100(1-\alpha)$ percent confidence interval for $\mu$ is given by

$$
\bar{Y}(n) \pm t_{n-1,1-\alpha / 2} \sqrt{\frac{S^{2}(n)}{n}}
$$

where $t_{n-1,1-\alpha / 2}$ is the upper $1-\alpha / 2$ critical point for the $t$ distribution with $n$ - 1 degrees of freedom and $S^{2}(n)$ is the sample variance given by 


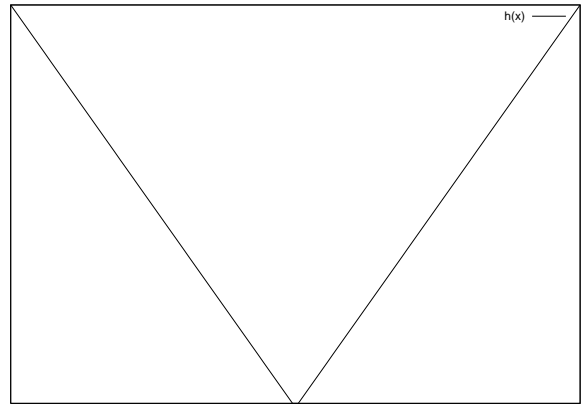

Fig. 1. Bimodal distribution

$$
S^{2}(n)=\frac{\sum_{j=1}^{n}\left(Y_{j}-\bar{Y}(n)\right)^{2}}{n-1} .
$$

Next, simulation results are obtained for other demand patterns than the compound Poisson with exponentially distributed order sizes. We distinguish three different types of distributions for the order sizes: a left triangular distribution with mode equal to $a$, a right triangular distribution with mode equal to $b$ and a bimodal distribution (see Figure 1. The results are compared to the results of the simulation model with exponentially distributed order sizes using confidence intervals.

When comparing two alternative systems, a confidence interval can be constructed for the difference in the two expectations. This does not only results in a "reject" or "fail-to-reject" conclusion but also quantifies how much the measures differ. When the number of replications for each alternative is the same $\left(n_{1}=n_{2}=n\right)$, a paired- $t$ confidence interval can be built. Let $Y_{j 1}$ and $Y_{j 2}$ be the corresponding outputs of two alternatives and define $Z_{j}=Y_{j 1}-Y_{j 2}$, for $j=1,2, \ldots, n$. Then $E\left(Z_{j}\right)=\nu$ is the quantity for which we want to construct a confidence interval. Assuming that $Z_{j}$ 's are iid random variables, let

$$
\bar{Z}(n)=\frac{\sum_{j=1}^{n} Z_{j}}{n}
$$

and

$$
S^{2}(n)=\frac{\sum_{j=1}^{n}\left(Z_{j}-\bar{Z}(n)\right)^{2}}{n-1},
$$

the approximate $100(1-\alpha)$ percent confidence interval is

$$
\bar{Z}(n) \pm t_{n-1,1-\alpha / 2} \sqrt{\frac{S^{2}(n)}{n}} .
$$

This is called the paired- $t$ confidence interval. If this interval does not contain zero, it can be concluded that the two responses are different.

\section{B. Common random numbers}

In this simulation study, we use multiple runs for each alternative using the same random numbers for the generation of the operating time until failure and the repair time of a failed machine for each corresponding pair of alternatives. This ensures that differences in the results are only due to a different demand process.

\section{Simulation model and Validation of the MODEL}

To test the robustness of the results of Posner and Berg (1989), we need to build a simulation model of the model of Posner and Berg. The simulation model is built in Arena (Rockwell Software). The parameters and initial conditions of the simulation model are given in Table I. The performance measures that are considered in this paper are: availability (fraction of time machine engaged in active production), fraction of time machine down due to failures, demand rate, fraction of demand satisfied and machine shutdown rate due to failures. The simulation is run for 3600 time-units with a warm-up period of 720 time-units and 20 replications are made.

\begin{tabular}{|l|c|}
\hline Parameter & Value \\
\hline Operating time until failure & $\operatorname{Expo}(1 / 9)$ \\
\hline Repair time & $\operatorname{Expo}(1 / 3)$ \\
\hline Demand arrivals & $\operatorname{Poisson}(0.3)$ \\
\hline Order sizes & $\operatorname{Expo}(1 / 3)$ \\
\hline Maximum inventory level & 100 \\
\hline Initial Inventory & 0 \\
\hline
\end{tabular}

TABLE I: Parameters and initial conditions of the simulation model

Validation is the process of determining whether a simulation model is an accurate representation of the system (Law, 2005). To validate the simulation model, the results are compared to the analytical results of Posner and Berg. However, since the model is only an approximation to the actual system, a null hypothesis that the system and model are the "same" is clearly false (Law, 2007). Law (2007) believes that it is more useful to ask whether or not the differences between the model and the system are significant enough to affect any conclusions derived from the model. In Table II the analytical results and the means and standard deviations of the simulation results over 20 replications are shown.

To validate the model, we first calculate a confidence interval for each performance measure. These $95 \%$ confidence intervals are shown in Table III and are used to decide whether or not the differences between the system and the model are small enough to have a valid model. For each performance measure, the confidence interval contains the analytical value calculated by Posner and Berg so we cannot demonstrate that there is a significant difference between the simulation results and the analytical result. Therefore, we cannot reject the null hypothesis that the simulation results are "equal" to the analytical results.

To demonstrate that there is not only no significant difference between the results but also that the results are close to each other, we calculated the probability 


\begin{tabular}{|l|c|c|c|}
\hline & \multirow{2}{*}{$\begin{array}{c}\text { Analytical } \\
\text { results }\end{array}$} & \multicolumn{2}{|c|}{ Simulation results } \\
\cline { 3 - 4 } & 0.7495 & 0.7448 & 0.013 \\
\hline Availability & 0.2498 & 0.2549 & 0.0134 \\
\hline $\begin{array}{l}\text { Fraction of time } \\
\text { machine down } \\
\text { to failures }\end{array}$ & & & \\
\hline Demand rate & 0.9 & 0.9029 & 0.0428 \\
\hline $\begin{array}{l}\text { Fraction of de- } \\
\text { mand satisfied }\end{array}$ & 0.8328 & 0.8259 & 0.0416 \\
\hline $\begin{array}{l}\text { Machine shut- } \\
\text { down rate due } \\
\text { to overstocking }\end{array}$ & 0.000191 & 0.000333 & 0.00149 \\
\hline
\end{tabular}

TABLE II: Comparison of analytical results with simulation results

\begin{tabular}{|l|c|c|}
\hline & LCL & UCL \\
\hline Availability & 0.739 & 0.751 \\
\hline $\begin{array}{l}\text { Fraction of time } \\
\text { machine down } \\
\text { to failures }\end{array}$ & 0.249 & 0.261 \\
\hline Demand rate & 0.883 & 0.923 \\
\hline $\begin{array}{l}\text { Fraction of de- } \\
\text { mand satisfied }\end{array}$ & 0.806 & 0.845 \\
\hline $\begin{array}{l}\text { Machine shut- } \\
\text { down rate due } \\
\text { to overstocking }\end{array}$ & 0 & 0.00103 \\
\hline
\end{tabular}

TABLE III: Confidence intervals for the simulation results

that the simulation model generates a value that is maximum 1, 3 or $5 \%$ error from the analytical result. These probabilities are given in Table IV. For each performance measure, there is at least a $75 \%$ probability that the simulation model generates a value that is in a range of $5 \%$ from the analytical result of Posner and Berg. For the availability, the fraction of time the machine is down due to failures or down due to overstocking, the probability is even above $99 \%$ which means the results of the simulation model are close to the analytical results.

\begin{tabular}{|l|c|c|c|}
\hline & $1 \%$ & $3 \%$ & $5 \%$ \\
\hline Availability & 52.73 & 96.97 & 99.97 \\
\hline $\begin{array}{l}\text { Fraction of time } \\
\text { machine down } \\
\text { to failures }\end{array}$ & 51.26 & 96.39 & 99.96 \\
\hline Demand rate & 18.42 & 51.53 & 75.59 \\
\hline $\begin{array}{l}\text { Fraction of de- } \\
\text { mand satisfied }\end{array}$ & 18.73 & 52.29 & 76.4 \\
\hline $\begin{array}{l}\text { Machine shut- } \\
\text { down rate due } \\
\text { to overstocking }\end{array}$ & 100 & 100 & 100 \\
\hline
\end{tabular}

TABLE IV: Probabilities that the simulated result is in a range of 1.3 or $5 \%$ from the analytical result
Based on these results, we can conclude that the simulation model is a good representation of the system and is therefore a valid simulation model.

\section{Demand process}

The objective of this paper is to test the robustness of the results of Posner and Berg to changes in the demand process. The demand process is compound Poisson and, with the parameters used in the previous section, has a mean of 0.9 units and a variance of 5.4.

In practice, information about the form of the probability distribution is often limited. For example, it might be that only the first two moments of the probability distribution are known. We assume the same mean and standard deviation for demand can be obtained by various patterns regarding demand frequency and size.

In this section, we relax the assumption that the probability distribution of the demand is completely known and merely assume that the first two moments are known and finite. Different order sizes are used in the simulation model but the parameters are chosen in such a way that they do not result in a different mean or standard deviation of the demand distribution.

When the mean $\mathrm{M}$ and variance $\mathrm{V}$ of the demand distribution are known, the compound Poisson distribution with exponentially-distributed order sizes has parameters:

$$
\lambda=\frac{2 M^{2}}{V}
$$

and

$$
\mu=\frac{\lambda}{M}
$$

Three other distributions for the order sizes, all in a range $[a, b]$, are used. When the order sizes follow a triangular distribution with mode $a$, the compound Poisson distribution has parameters:

$$
\frac{M^{2}}{V} \leq \lambda \leq \frac{3 M^{2}}{2 V}
$$

and

$$
\begin{array}{r}
a=\frac{M-\sqrt{2 \lambda V-2 M^{2}}}{\lambda} \\
b=\frac{M+2 \sqrt{2 \lambda V-2 M^{2}}}{\lambda} .
\end{array}
$$

When the order sizes are triangular with mode $b$, the compound Poisson distribution has parameters:

$$
\frac{M^{2}}{V} \leq \lambda \leq \frac{9 M^{2}}{8 V}
$$

and

$$
\begin{aligned}
a & =\frac{M-2 \sqrt{2 \lambda V-2 M^{2}}}{\lambda} \\
b & =\frac{M+\sqrt{2 \lambda V-2 M^{2}}}{\lambda} .
\end{aligned}
$$


When the order sizes follow a bimodal distribution with modes $a$ and $b$, the compound Poisson distribution has parameters:

$$
\frac{M^{2}}{V} \leq \lambda \leq \frac{3 M^{2}}{2 V}
$$

and

$$
\begin{aligned}
& a=\frac{M-\sqrt{2 \lambda V-2 M^{2}}}{\lambda} \\
& b=\frac{M+\sqrt{2 \lambda V-2 M^{2}}}{\lambda} .
\end{aligned}
$$

When $M=0.9$ and $V=5.4$, the results for the parameters are given in Table $\mathrm{V}$.

\begin{tabular}{|c|c|c|c|c|}
\hline & Exponential & $\begin{array}{c}\text { Left } \\
\text { triangular }\end{array}$ & $\begin{array}{c}\text { Right } \\
\text { triangular }\end{array}$ & Bimodal \\
\hline$\lambda$ & 0.3 & 0.16 & 0.16 & 0.16 \\
\hline$\mu$ & 0.33 & & & \\
\hline$a$ & & 3.57 & 1.52 & 3.57 \\
\hline$b$ & & 9.73 & 7.68 & 7.67 \\
\hline
\end{tabular}

TABLE V: Parameters of the demand process

\section{Results}

The simulation model uses common random numbers for the generation of the operating time until failure and the repair time of a failed machine. This ensures that differences in the results are due only to a different demand process. For each different demand process, 20 replications of 3600 time-units (with a warm-up period of 720 time-units) are made. The means of the simulation results for each of the performance measures are given in Table VI.

To test the robustness of the analytical results of Posner and Berg to changes in the demand process, $95 \%$ confidence intervals are constructed for every performance measure. The confidence intervals for comparing the triangular distribution with mode $a$ to the exponential distribution can be found in Table VII. The confidence intervals for the difference between the results of the simulation model with a triangular distribution with mode $b$ and the results of the model with an exponential distribution are given in Table VIII. Table IX shows the confidence intervals for comparing the bimodal distribution to the exponential distribution.

All the confidence intervals in Table VII, Table VIII and Table IX contain 0 . This means that the difference between the results of the simulation model with an exponentially-distributed order size and the results of the simulation model with a different distribution for the order sizes is not significantly different from zero for each of the performance measures. Changes in the demand process have no significant impact on performance measures for the production-inventory system described in this paper. The model of Posner and Berg is a robust model. The analytical results obtained by the model give a good indication for the performance

\begin{tabular}{|l|c|c|}
\hline & LCL & UCL \\
\hline Availability & -0.000941 & 0.000976 \\
\hline $\begin{array}{l}\text { Fraction of time } \\
\text { machine down } \\
\text { to failures }\end{array}$ & -7.07 & $2.73 \mathrm{E}-5$ \\
\hline Demand rate & -0.0362 & 0.0127 \\
\hline $\begin{array}{l}\text { Fraction of de- } \\
\text { mand satisfied }\end{array}$ & -0.0112 & 0.0316 \\
\hline $\begin{array}{l}\text { Machine shut- } \\
\text { down rate due } \\
\text { to overstocking }\end{array}$ & -0.000992 & 0.001 \\
\hline
\end{tabular}

TABLE VII: Confidence intervals for comparing exponential with left triangular results

\begin{tabular}{|l|c|c|}
\hline & LCL & UCL \\
\hline Availability & -0.000946 & 0.00113 \\
\hline $\begin{array}{l}\text { Fraction of time } \\
\text { machine down } \\
\text { to failures }\end{array}$ & -7.07 & $2.73 \mathrm{E}-5$ \\
\hline Demand rate & -0.0385 & 0.0114 \\
\hline $\begin{array}{l}\text { Fraction of de- } \\
\text { mand satisfied }\end{array}$ & -0.00973 & 0.0339 \\
\hline $\begin{array}{l}\text { Machine shut- } \\
\text { down rate due } \\
\text { to overstocking }\end{array}$ & -0.00114 & 0.001 \\
\hline
\end{tabular}

TABLE VIII: Confidence intervals for comparing exponential with right triangular results

measures of a similar production-inventory system facing another demand process.

\section{CONCLUSIONS AND FURTHER RESEARCH}

In this paper, we test the robustness of analytical results on performance measures for a productioninventory system with an unreliable production facility. These results are calculated for the special case that the demand process is compound Poisson with exponentially-distributed order sizes. A simulation model is used to obtain the same performance measures for this production-inventory system facing another demand pattern. Three different demand patterns are compared: compound Poisson with order sizes that follow a triangular distribution with mode $a$, a triangular distribution with mode $b$ or a bimodal distribution. The parameters of these demand patterns are chosen so that they do not cause a change of the mean and variance of the aggregate demand distribution.

The performance measures for these three different demand patterns do not differ significantly from the closed-form performance measures obtained by Posner and Berg for the special case of an exponentiallydistributed order size. Their results are therefore robust to changes in the demand pattern.

In further research, other distributions for the order sizes can be used. The simulation model can also be used to determine the optimal value of the pre-set 


\begin{tabular}{|l|c|c|c|c|}
\hline & Exponential & $\begin{array}{c}\text { Left } \\
\text { triangular }\end{array}$ & $\begin{array}{c}\text { Right } \\
\text { triangular }\end{array}$ & Bimodal \\
\hline Availability & 0.7448 & 0.7448 & 0.7447 & 0.7449 \\
\hline Fraction of time machine down to failures & 0.2549 & 0.2549 & 0.259523 & 0.2547 \\
\hline Demand rate & 0.9029 & 0.9147 & 0.9164 & 0.9228 \\
\hline Fraction of demand satisfied & 0.8259 & 0.8157 & 0.8138 & 0.8101 \\
\hline Machine shutdown rate due to overstocking & 0.000333 & 0.000328 & 0.000401 & 0.000346 \\
\hline
\end{tabular}

TABLE VI: Results for the different demand processes

\begin{tabular}{|l|c|c|}
\hline & LCL & UCL \\
\hline Availability & -0.000896 & 0.000643 \\
\hline $\begin{array}{l}\text { Fraction of time } \\
\text { machine down } \\
\text { to failures }\end{array}$ & -0.000207 & 0.000486 \\
\hline Demand rate & -0.0503 & 0.0105 \\
\hline $\begin{array}{l}\text { Fraction of de- } \\
\text { mand satisfied }\end{array}$ & -0.0113 & 0.0428 \\
\hline $\begin{array}{l}\text { Machine shut- } \\
\text { down rate due } \\
\text { to overstocking }\end{array}$ & -000104 & 0.00102 \\
\hline
\end{tabular}

TABLE IX: Confidence intervals for comparing exponential with bimodal results

threshold or the maximum inventory level. Furthermore, the simulation model can be extended to more machines in series, separated by finite buffers. Srensen and Janssens (2003) calculate customer service levels and other performance measures as a function of the availabilities of the machines and the sizes of intermediate buffers for a model with $n$ machines in series, separated by finite buffers.

\section{REFERENCES}

[1] Brill, P. and M. Posner. 1977. "Level crossings in point processes applied to queues: Single server case." Operations Research, 25, pp. 662-674.

[2] Brill, P. and M. Posner. 1981. "The system point method in exponential queues: A level crossing approach." Mathematics of Operations Research, 6, pp. 31-49.

[3] Kelton, W.D.; R.P. Sadowski and D.T. Sturrock. 2007. Simulation with Arena, 4th edition, McGraw-Hill, Singapore.

[3] Law, A.M. 2007. Simulation Modeling and Analysis, 4th edition, McGraw-Hill International Editions, Singapore.

[4] Law, A.M. 2005. "How to build valid and credible simulation models." Proceedings of the 2005 Winter Simulation Conference, Orlando, 4-7 December 2007, pp. 24-32.

[5] Posner, M. and M. Berg. 1989. "Analysis of a productioninventory system with unreliable production facility." Operations Research Letters 8, pp. 339-345.

[6] Sörensen, K. and G.K. Janssens. 2003. "A productioninventory system with an unreliable continuous transfer line." Journal of Systems Science and Systems Engineering 12(3), pp. 298-306.

\section{BIOGRAPHY}

Katrien Ramaekers graduated as Master of Business Economics, option Technology at the Limburg University Centre in 2002. In October 2002, she started as a Ph.D.-student at Hasselt University. Her main research interest is on the integration of simulation and optimisation, especially as a support for complex logistics decision-making and for decision support with limited information in supply and demand. She is a member of the Data Analysis and Modelling research group and of the Transportation Research Institute of Hasselt University.

Gerrit K. Janssens received degrees of M.Sc. in Engineering with Economy from the University of Antwerp (RUCA), Belgium, M.Sc. in Computer Science from the University of Ghent (RUG), Belgium, and Ph.D. from the Free University of Brussels (VUB), Belgium. After some years of work at General Motors Continental, Antwerp, he joined the University of Antwerp until the year 2000. Currently he is Professor of Operations Management and Logistics at Hasselt University within the Faculty of Business Administration. He also holds the CPIM certificate of the American Production and Inventory Control Society (APICS). During the last fifteen years he has repeatedly been visiting faculty member of universities in Thailand, Vietnam, Philippines, Cambodia and Zimbabwe. His main research interests include the development and application of operations research models in production and distribution logistics. 